\title{
ON SYMMETRIC QUASICIRCLES
}

\author{
SHENGJIAN WU and SHANSHUANG YANG
}

(Received 6 November 1998; revised 22 June 1999)

Communicated by P. G. Fenton

\begin{abstract}
We study an important subclass of quasicircles, namely, symmetric quasicircles. Several characterizations for quasicircles, such as the reverse triangle inequality, the $M$-condition and the quasiconformal extension property, have been extended to symmetric quasicircles by Becker and Pommerenke and by Gardiner and Sullivan. In this paper we establish several relations among various domain constants such as quasiextremal distance constants, (local) reflection constants and (local) extension constants for this class. We also give several characterizations for symmetric quasicircles such as the strong quadrilateral inequality and the strong extremal distance property. They correspond to the quadrilateral inequality and the extremal distance property for quasicircles.

1991 Mathematics subject classification (Amer. Math. Soc.): primary 30C62, 31Bxx.

Keywords and phrases: quasicircle, symmetric, quasisymmetric, quasiextremal distance, quasiconformal, reflection, extension, modulus, dilatation.
\end{abstract}

\section{Introduction}

A Jordan curve $J$ in the plane $\mathbb{C}$ is said to be a symmetric quasicircle if

$$
\max _{w \in J(a, b)} \frac{|a-w|+|w-b|}{|a-b|} \rightarrow 1
$$

as $a, b \in J$ and $|a-b| \rightarrow 0$, where $J(a, b)$ is the smaller arc of $J$ between $a$ and $b$. This class of quasicircles was introduced by Becker and Pommerenke [BP] as asymptotically conformal curves. They gave a number of analytical characterizations for symmetric quasicircles in terms of conformal mappings (see [Po, Theorem 11.1]). Later Gardiner and Sullivan [GS] introduced the corresponding concept of symmetric

This research was supported in part by the NNSF of China and by the NSF of US.

(C) 2000 Australian Mathematical Society 0263-6115/2000\$A2.00+0.00 
homeomorphisms of the unit circle (or real line) from a topological point of view. They defined the group $S$ of symmetric homeomorphisms as a natural topological subgroup of the group $Q S$ of quasisymmetric homeomorphisms under the uniform topology, and discussed a variety of analytical and geometrical properties of symmetric homeomorphisms and symmetric quasicircles.

It is well known that quasisymmetric homeomorphisms are boundary values of quasiconformal mappings and they can be characterized by the following so called $M$-condition. A homeomorphism $h$ of the unit circle $S^{1}$ is said to satisfy the $M$-condition if there is a constant $M \geq 1$ such that

$$
\frac{1}{M} \leq \frac{|h(c)-h(b)|}{|h(b)-h(a)|} \leq M
$$

for any symmetrically located triples $a, b, c$ on the circle. As proved by Gardiner and Sullivan [GS, Theorem 2.1], symmetric homeomorphisms can be characterized by the following strong $M$-condition: for any $\epsilon>0$, there exists $\delta>0$ such that

$$
\frac{1}{1+\epsilon} \leq \frac{|h(c)-h(b)|}{|h(b)-h(a)|} \leq 1+\epsilon
$$

for any symmetrically located triples $a, b, c$ within a $\delta$-neighborhood of each other. Symmetric quasicircles have a similar characterization.

Let $J$ be a bounded Jordan curve in the complex plane $\mathbb{C}$. Denote its interior and exterior domains by $\Omega$ and $\Omega^{*}$, respectively. Let $f$ and $g$ be Riemann mappings which map the unit disk $D$ onto $\Omega$ and the exterior $D^{*}$ onto $\Omega^{*}$, respectively. Extend $f$ and $g$ homeomorphically to the boundary and define a homeomorphism $h$ of $S^{1}$ by $h=g^{-1} \circ f$. Then $J$ is a quasicircle or symmetric quasicircle if and only if $h$ is quasisymmetric or symmetric, respectively. We call $h$ a homeomorphism induced by $J$. Just like quasicircles can be characterized by a reverse triangle inequality, symmetric quasicircles can be characterized by a strong reverse triangle inequality ([BP] and [GS, Theorem 6.1]). All smooth Jordan curves are symmetric quasicircles and symmetric quasicircles do not allow corners. But a symmetric quasicircle can still be very wild in the sense that it does not possess a tangent almost everywhere in the linear measure [Po, page 249].

From the above and other characterizations given in [Po] and [GS] one can see that the difference between a symmetric quasicircle and a nonsymmetric quasicircle is detected only on fine scales. In this paper we give two more characterizations for symmetric quasicircles, the strong quadrilateral inequality and the strong extremal distance property (Theorem 4.1). These correspond to the quadrilateral inequality and the extremal distance property for quasicircles (see [Ge, III.D.4 and III.E.4]). The idea is to replace the appropriate constant by a constant that is closer and closer to the constant corresponding to the circle as the scale gets smaller and smaller. 
In Section 2 we investigate the relations among various domain constants such as the QED constant, reflection constant and quasicircle constant for symmetric quasicircles. We show that a conjecture by Garnett and Yang [GY] does not hold for this class of Jordan curves.

\section{Domain constants and their relations}

For a Jordan domain $\Omega$ its quasiextremal distance constant (or QED constant) with respect to the boundary, denoted by $M_{b}(\Omega)$, is defined as

$$
M_{b}(\Omega)=\sup _{A, B} \frac{\bmod (A, B ; \mathbb{C})}{\bmod (A, B ; \Omega)},
$$

where the supremum is taken over all pairs of disjoint nondegenerate continua $A$ and $B$ on the boundary $\partial \Omega$, and $\bmod (A, B ; \Omega)$ denotes the modulus of the family $\Gamma(A, B ; \Omega)$ of curves that join $A$ and $B$ in $\Omega$. The quasiconformal reflection constant (or reflection constant) of $\Omega$, denoted by $R(\Omega)$, is defined as

$$
R(\Omega)=\inf _{f} K(f)
$$

where the infimum is taken over all homeomorphic reflections $f$ in the boundary $\partial \Omega$ and $K(f)$ denotes the maximal dilatation of $f$. A homeomorphic reflection in a Jordan curve is a homeomorphism of $\overline{\mathbb{C}}$ that interchanges the two components of the complement of the curve taken with respect to the extended plane and fixes the curve pointwise. For more information on the modulus of a curve family and maximal dilatations of quasiconformal mappings, we refer the reader to [LV] or any other book on the theory of quasiconformal mappings in the plane.

It is well known that both $M_{b}(\Omega)$ and $R(\Omega)$ are invariant under Möbius transformations and that their values reflect, to some extent, the geometry of a domain. For example, the following three statements are equivalent: $\Omega$ is a disk or a half plane; $M_{b}(\Omega)=2 ; R(\Omega)=1$. It is also true that $M_{b}(\Omega) \leq R(\Omega)+1$ for any Jordan domain $\Omega$. For more details and related results we refer the reader to [GY, Y3] and the references therein. Furthermore, based on observations in some special cases, it was conjectured in [GY] that $M_{b}(\Omega)=R(\Omega)+1$ for any Jordan domain $\Omega$. However, after delicate analysis and estimates, we have recently shown that this conjectured relation is not true for smooth Jordan domains [Y3]. In this paper, we further show that this conjecture is false for all symmetric quasicircles other than circles and straight lines. But we need to point out that the QED constant $M(\Omega)$ considered in [GY, Y3] is somewhat different from the one considered here and is defined by taking the supremum over all pairs of disjoint nondegenerate continua $A, B \subset \bar{\Omega}$ in (2.1) above. 
THEOREM 2.1. For any Jordan domain $\Omega$ whose boundary is a symmetric quasicircle, other than disks and half planes, we have

$$
M_{b}(\Omega)<R(\Omega)+1 .
$$

Similar to the reflection constant, we define the quasicircle constant $K(\Omega)$ of $\Omega$ as the infimum of the maximal dilatations of all quasiconformal maps $f$ of the plane that map $\partial \Omega$ onto the unit circle. Kühnau [Ku] proved that $R(\Omega)=K(\Omega)^{2}$ for any $\Omega$. Because the difference between a symmetric quasicircle and a nonsymmetric quasicircle is only detected through a fine scale, we also consider the local versions of the reflection and quasicircle constants. We let $H(\Omega)$ denote the local reflection constant which is defined as in (2.2) with the maximal dilatation $K(f)$ of $f$ being replaced by the local maximal dilatation of $f$ in arbitrarily small neighborhoods of the boundary. Note that $H(\Omega)$ is exactly the boundary dilatation of the homeomorphism of the unit circle induced by the boundary $\partial \Omega$ as defined by Fehlmann [Fe] (see also [GS, Definition 3.3]). Corresponding to the quasicircle constant, we define the local quasicircle constant $H_{1}(\Omega)$ by replacing the global maximal dilatations with the local ones in arbitrarily small neighborhoods of $\partial \Omega$. We establish the following relation between $H(\Omega)$ and $H_{1}(\Omega)$.

THEOREM 2.2. For any Jordan domain $\Omega$ we have

$$
H(\Omega)=H_{1}(\Omega)^{2} .
$$

Proof. We first show that $H \leq H_{1}^{2}$. By the definition of $H_{1}$, for any $\epsilon>0$ there is a QC mapping $f: \hat{\mathbb{C}} \rightarrow \hat{\mathbb{C}}$ that maps the unit circle $\partial D$ onto $\partial \Omega$ with dilatation less than or equal to $H_{1}+\epsilon$ in a neighborhood of $\partial D$. Set

$$
F(z)=f \circ j \circ f^{-1}(z)
$$

where $j$ is the conformal reflection about $\partial D$. Then $F$ is a reflection about $\partial \Omega$ with dilatation not greater than $\left(H_{1}+\epsilon\right)^{2}$ in a neighborhood of $\partial \Omega$. This shows that $H \leq H_{1}^{2}$.

To show that $H_{1}^{2} \leq H$, let $f: \hat{\mathbb{C}} \rightarrow \hat{\mathbb{C}}$ be any QC mapping with $f: D \rightarrow \Omega$ being quasiconformal and $f: D^{*} \rightarrow \Omega^{*}$ being conformal. Let $\mu(z)$ denote the complex dilatation of $f$ and $K_{f}(z)$ the local maximal dilatation at $z$ :

$$
K_{f}(z)=\frac{1+|\mu(z)|}{1-|\mu(z)|}
$$

Set $\mu_{1}(z)=t(z) \mu(z)$, where

$$
t(z)=\frac{K_{f}(z)+1}{\left(K_{f}^{1 / 2}(z)+1\right)^{2}} .
$$


By solving a Beltrami equation (and composing the solution with a conformal map if necessary), one can construct QC map $g_{1}: D \rightarrow D$ with complex dilatation $\mu_{1}(z)$.

Let $g_{2}=f \circ g_{1}^{-1}: D \rightarrow \Omega$. Direct computations show that

$$
K_{g_{1}}(z)=\frac{1+\left|\mu_{1}(z)\right|}{1-\left|\mu_{1}(z)\right|}=K_{f}^{1 / 2}(z)
$$

and that

$$
\left|\mu_{g_{2}}\left(g_{1}(z)\right)\right|=\frac{\left|\mu(z)-\mu_{1}(z)\right|}{\left|1-\bar{\mu}_{1}(z) \mu(z)\right|}=\frac{(1-t(z))|\mu(z)|}{1-t(z)|\mu(z)|^{2}} .
$$

Thus it follows that

$$
K_{g_{2}}\left(g_{1}(z)\right)=K_{f}^{1 / 2}(z)
$$

Finally, let

$$
F(w)= \begin{cases}g_{2}(w), & w \in \bar{D} \\ f \circ j \circ f^{-1} \circ g_{2} \circ j(w), & w \in D^{*} .\end{cases}
$$

Then $F: \hat{\mathbb{C}} \rightarrow \hat{\mathbb{C}}$ is a QC map with $F(D)=\Omega$. Furthermore, since $f$ is conformal in $D^{*}$, it follows that

$$
K_{F}(w)= \begin{cases}K_{g_{2}}=K_{f}^{1 / 2}\left(g_{\uparrow}^{-1}(w)\right), & w \in D, \\ K_{f^{-}{ }^{\circ} \circ g_{2}}=K_{g_{1}}=K_{f}^{1 / 2}\left(g_{1}^{-1} \circ j(w)\right), & w \in D^{*} .\end{cases}
$$

In particular, if the above QC map $f$ has maximal dilatation less than or equal to $H(\Omega)+\epsilon$ in a neighborhood of $\partial D$, then the maximal dilatation of $F$ is less than or equal to $(H(\Omega)+\epsilon)^{1 / 2}$. This shows that $H_{1} \leq(H+\epsilon)^{1 / 2}$ for any $\epsilon>0$. Thus it follows that $H_{1}^{2} \leq H$ as desired.

REMARK. The above argument also proves the identity that $R(\Omega)=K(\Omega)^{2}$ for the global reflection and quasicircle constants which was first observed by Kühnau [Ku].

In order to prove Theorem 2.1 we need the following refinement of the quasiinvariance of moduli under quasiconformal mappings. For a doubly connected domain $R$ we let $\bmod (R)$ denote the modulus of the curve family that joins the two complimentary components of $R$.

LEMMA 2.3. Let $R_{1}$ and $R_{2}$ be two doubly connected domains and let $f: R_{1} \rightarrow R_{2}$ be a quasiconformal map. Then

$$
\max \left\{\frac{\bmod \left(R_{1}\right)}{\bmod \left(R_{2}\right)}, \frac{\bmod \left(R_{2}\right)}{\bmod \left(R_{1}\right)}\right\} \leq C \iint_{R_{1}} K_{f}(z) \frac{\left|g^{\prime}(z)\right|^{2}}{|g(z)|^{2}} d x d y,
$$


where $w=g(z)$ is a conformal map of $R_{1}$ onto an annulus $1<|w|<r$ and $C$ is a normalization constant so that

$$
C \iint_{R_{1}} \frac{\left|g^{\prime}(z)\right|^{2}}{|g(z)|^{2}} d x d y=1 .
$$

This result follows directly from Grötzsch's length-area argument (see [Ga, page 26, (29)-(30)]). In particular, if one maps the annulus $1<|w|<r$ onto a rectangle by the logarithmic function and let $R_{1}$ be the doubly connected domain whose complement consists of two finite intervals $[a, b]$ and $[c, d]$ on the real axis, then from Lemma 2.3 one can deduce that

$$
\max \left\{\frac{\bmod \left(R_{1}\right)}{\bmod \left(R_{2}\right)}, \frac{\bmod \left(R_{2}\right)}{\bmod \left(R_{1}\right)}\right\} \leq \iint_{R_{1}} K_{f}(z)\left|\phi^{\prime}(z)\right|^{2} d x d y,
$$

where

$$
\phi^{\prime}(z)^{2}=\frac{C(a, b, c, d)}{(z-a)(z-b)(z-c)(z-d)}
$$

and $C(a, b, c, d)$ is the normalization constant.

\section{Proof of Theorem 2.1}

For the proof of Theorem 2.1, we let $\Omega$ be a bounded Jordan domain other than a disk. For each $n \geq 1$, fix disjoint nondegenerate continua $A_{n}$ and $B_{n}$ on the boundary $\partial \Omega$ such that

$$
M_{b}(\Omega)=\lim _{n \rightarrow \infty} \frac{\bmod \left(A_{n}, B_{n} ; \mathbb{C}\right)}{\bmod \left(A_{n}, B_{n} ; \Omega\right)}
$$

and that $A_{n}$ and $B_{n}$ converge in the Hausdorff metric to continua $A$ and $B$, respectively.

We consider first the nondegenerate case, namely, the case when the limit sets $A$ and $B$ are disjoint nondegenerate continua. In this case, by the continuity of moduli (see, for example, [He, Theorem 3.3 and Theorem 5.9]), we have

$$
M_{b}(\Omega)=\frac{\bmod (A, B ; \mathbb{C})}{\bmod (A, B ; \Omega)}
$$

Then an argument using capacity and harmonic functions (see [Y3, Section 3]) shows that

$$
\frac{\bmod \left(A, B ; \Omega^{*}\right)}{\bmod (A, B ; \Omega)}=R(\Omega)
$$


if (2.3) does not hold (namely, if $M_{b}(\Omega)=1+R(\Omega)$ ). On the other hand, let $h$ be a homeomorphism of the unit circle induced by $\partial \Omega$ as defined in the introduction. If $\partial \Omega$ is a symmetric quasicircle, then it follows from [Y2, Corollary 2.6] that

$$
\sup _{A, B \subset \partial \Omega} \frac{\bmod \left(A, B ; \Omega^{*}\right)}{\bmod (A, B ; \Omega)}=K_{h}<K_{h}^{*}=R(\Omega) .
$$

This contradicts (3.3) and shows that (2.3) holds in the nondegenerate case.

Depending on the size and relative positions of the limit sets $A$ and $B$, there are three degenerate cases to be considered.

Case 1. $A, B$ both are nondegenerate and $A \cap B \neq \emptyset$;

Case 2. At least one of the two sets $A, B$ is a single point and $A \cap B=\emptyset$;

Case 3. At least one of the two sets $A, B$ is a single point and $A \cap B \neq \emptyset$.

In all these cases we will show that

$$
M_{b}(\Omega) \leq 2 H,
$$

where $H$ is the local reflection constant of $\Omega$ defined in the previous section. Fix $\epsilon>0$. By the definition of $H$, there is a quasiconformal map $f: \mathbb{C} \rightarrow \mathbb{C}$ such that $f: \Omega \rightarrow D$ is conformal and that

$$
K(f) \leq H+\epsilon
$$

in a Jordan domain $\Omega_{\epsilon}$ that contains the closure of $\Omega$.

We shall deal with Case 1 first sincè it is the easiest. Using some basic properties of the modulus, one can easily deduce that

$$
\begin{aligned}
\bmod \left(A_{n}, B_{n} ; \Omega_{\epsilon}\right) & \leq(H+\epsilon) \bmod \left(A_{n}^{\prime}, B_{n}^{\prime} ; f\left(\Omega_{\epsilon}\right)\right) \\
& \leq 2(H+\epsilon) \bmod \left(A_{n}^{\prime}, B_{n}^{\prime} ; D\right)=2(H+\epsilon) \bmod \left(A_{n}, B_{n} ; \Omega\right)
\end{aligned}
$$

and hence that

$$
\begin{aligned}
\bmod \left(A_{n}, B_{n} ; \mathbb{C}\right) & \leq \bmod \left(A_{n}, B_{n} ; \Omega_{\epsilon}\right)+\bmod \left(\partial \Omega, \partial \Omega_{\epsilon} ; \mathbb{C}\right) \\
& \leq 2(H+\epsilon) \bmod \left(A_{n}, B_{n} ; \Omega\right)+\bmod \left(\partial \Omega, \partial \Omega_{\epsilon} ; \mathbb{C}\right) .
\end{aligned}
$$

We observe in this case that $\bmod \left(A_{n}, B_{n} ; \Omega\right) \rightarrow \infty$ and that $\bmod \left(\partial \Omega, \partial \Omega_{\epsilon} ; \mathbb{C}\right)$ is finite. Thus (3.6) yields that $M_{b}(\Omega) \leq 2(H+\epsilon)$ and (3.4) follows by letting $\epsilon \rightarrow 0$.

To deal with Case 2 , we need the following equivalent definition for the modulus due to Bagby [Ba].

Lemma 3.1 ([Ba, Theorem 5]). Let $A, B$ be two nondegenerate continua in the plane. For each $n \geq 1$ let

$$
W_{n}=\inf \left(\begin{array}{l}
n \\
2
\end{array}\right)^{-1} \sum_{1 \leq i<j \leq n} \log \left[a_{i}, a_{j}, b_{i}, b_{j}\right],
$$


where the infimum is taken over all choices of distinct points $a_{1}, \ldots, a_{n} \in A$ and $b_{1}, \ldots, b_{n} \in B$, and $\left[a_{i}, a_{j}, b_{i}, b_{j}\right]$ is the absolute cross ratio defined as

$$
\left[a_{i}, a_{j}, b_{i}, b_{j}\right]=\frac{\left|a_{i}-b_{j}\right|\left|a_{j}-b_{i}\right|}{\left|a_{i}-a_{j}\right|\left|b_{i}-b_{j}\right|} .
$$

Then

$$
\frac{2 \pi}{\bmod (A, B ; \mathbb{C})}=\lim _{n \rightarrow \infty} W_{n}
$$

To establish (3.4) in Case 2, we observe that

$$
\bmod \left(A_{n}, B_{n} ; \Omega\right)=\bmod \left(A_{n}^{\prime}, B_{n}^{\prime} ; D\right)=\frac{1}{2} \bmod \left(A_{n}^{\prime}, B_{n}^{\prime} ; \mathbb{C}\right) .
$$

Thus it follows that

$$
\frac{\bmod \left(A_{n}, B_{n} ; \mathbb{C}\right)}{\bmod \left(A_{n}, B_{n} ; \Omega\right)} \leq 2 \frac{\bmod \left(A_{n}, B_{n} ; \mathbb{C}\right)}{\bmod \left(A_{n}^{\prime}, B_{n}^{\prime} ; \mathbb{C}\right)}
$$

We shall show that

$$
\lim _{n \rightarrow \infty} \frac{\bmod \left(A_{n}, B_{n} ; \mathbb{C}\right)}{\bmod \left(A_{n}^{\prime}, B_{n}^{\prime} ; \mathbb{C}\right)} \leq(H+\epsilon)
$$

We will use Lemma 3.1 to estimate the left side of (3.8). By the Hölder continuity of quasiconformal maps, we have that

$$
|f(x)-f(y)| \leq L|x-y|^{1 /(H+\epsilon)}
$$

for all $x, y \in \partial \Omega$, where $L$ is a constant. Fix large $n$. For any sequences of distinct points $a_{1}, \ldots, a_{k} \in A_{n}$ and $b_{1}, \ldots, b_{k} \in B_{n}$, let $a_{i}^{\prime}=f\left(a_{i}\right)$ and $b_{i}^{\prime}=f\left(b_{i}\right)$ $(i=1, \ldots, k)$. Then, by (3.9) and the assumption that $A \cap B=\emptyset$, it follows that

$$
\left[a_{i}^{\prime}, a_{j}^{\prime}, b_{i}^{\prime}, b_{j}^{\prime}\right]=\frac{\left|a_{i}^{\prime}-b_{j}^{\prime}\right|\left|a_{j}^{\prime}-b_{i}^{\prime}\right|}{\left|a_{i}^{\prime}-a_{j}^{\prime}\right|\left|b_{i}^{\prime}-b_{j}^{\prime}\right|} \leq L_{1}\left[a_{i}, a_{j}, b_{i}, b_{j}\right]^{H+\epsilon}
$$

for $i \neq j$, where $L_{1}$ is a constant independent of $n, i, j$. Therefore,

$$
\begin{aligned}
W_{k}^{\prime} & =\inf \left(\begin{array}{l}
n \\
2
\end{array}\right)^{-1} \sum_{1 \leq i<j \leq k} \log \left[a_{i}^{\prime}, a_{j}^{\prime}, b_{i}^{\prime}, b_{j}^{\prime}\right] \\
& \leq \inf \left(\begin{array}{l}
n \\
2
\end{array}\right)^{-1} \sum_{1 \leq i<j \leq k}\left(\log L_{1}+(H+\epsilon) \log \left[a_{i}, a_{j}, b_{i}, b_{j}\right]\right) \\
& =\log L_{1}+(H+\epsilon) W_{n} .
\end{aligned}
$$


By Lemma 3.1, letting $k \rightarrow \infty$ yields that

$$
\frac{2 \pi}{\bmod \left(A_{n}^{\prime}, B_{n}^{\prime} ; \mathbb{C}\right)} \leq \log L_{1}+\frac{2 \pi(H+\epsilon)}{\bmod \left(A_{n}, B_{n} ; \mathbb{C}\right)} .
$$

We obvserve that $\bmod \left(A_{n}, B_{n} ; \mathbb{C}\right) \rightarrow 0$ as $n \rightarrow \infty$ since $A \cap B=\emptyset$. This yields (3.8) by multiplying by $\bmod \left(A_{n}, B_{n} ; \mathbb{C}\right)$ and letting $n$ approach infinity in (3.10). Thus (3.4) follows from (3.7) and (3.8) as desired in Case 2.

Next we deal with Case 3. In this case we will also show that (3.8) holds. In order to apply Lemma 2.3 and (2.8), we replace the unit disk by the upper halfplane and $A_{n}^{\prime}, B_{n}^{\prime}$ by finite intervals $A_{n}^{\prime}=\left[a_{n}^{\prime}, b_{n}^{\prime}\right], B_{n}^{\prime}=\left[c_{n}^{\prime}, d_{n}^{\prime}\right]$ on the real axis such that $a_{n}^{\prime}, b_{n}^{\prime}$ and $c_{n}^{\prime}$ all approach zero as $n \rightarrow \infty$. Then by (2.8) we have that

$$
\frac{\bmod \left(A_{n}, B_{n} ; \mathbb{C}\right)}{\bmod \left(A_{n}^{\prime}, B_{n}^{\prime} ; \mathbb{C}\right)} \leq \iint_{\mathbb{C}} K_{f^{-1}}(z)\left|\phi^{\prime}(z)\right|^{2} d x d y,
$$

where

$$
\phi^{\prime}(z)^{2}=\frac{C_{n}}{\left(z-a_{n}^{\prime}\right)\left(z-b_{n}^{\prime}\right)\left(z-c_{n}^{\prime}\right)\left(z-d_{n}^{\prime}\right)}
$$

and $C_{n}$ is the normalization constant such that the total mass of $\left|\phi^{\prime}(z)\right|^{2}$ is equal to 1 . It is easy to see that $C_{n} \rightarrow 0$ as $n \rightarrow \infty$. Fix a neighborhood $N_{\epsilon}$ of the origin such that $K_{f^{-1}}(z) \leq H+\epsilon$ when $z \in N_{\epsilon}$. Bỳ breaking the integral into two parts, one can deduce from (3.11) that

$$
\frac{\bmod \left(A_{n}, B_{n} ; \mathbb{C}\right)}{\bmod \left(A_{n}^{\prime}, B_{n}^{\prime} ; \mathbb{C}\right)} \leq(H+\epsilon)+\lambda C_{n},
$$

where $\lambda$ is a constant. Letting $n \rightarrow \infty$ yields (3.8), and hence (3.4) follows.

Finally, we assume that the boundary of $\Omega$ is a symmetric quasicircle. By [GS, Proposition 3.2], $H=1$. Thus (3.4) yields that $M_{b}(\Omega) \leq 2$ in all the degenerate cases. On the other hand, if $\Omega$ is not a disk or halfplane, then $R(\Omega)>1$. Thus (2.3) follows and this completes the proof of Theorem 2.1.

\section{Characterizations of symmetric quasicircles}

There are many different characterizations for quasicircles. For the most up to date list, we refer the reader to Gehring's recent survey paper [Ge]. Several characterizations, such as the reverse triangle inequality, the $M$-condition and the quasiconformal extension property, have been extended to symmetric quasicircles by Becker and Pommerenke [BP, Po] and by Gardiner and Sullivan [GS]. Here we obtain two more 
characterizations for symmetric quasicircles by extending two corresponding properties for quasicircles and by using the results from previous sections. The main idea in such an extension is to replace the appropriate constant for quasicircles by a constant that is closer and closer to the constant for a circle as the scale gets smaller and smaller.

First we recall two important characterizations for quasicircles. A quadrilateral $Q=\Omega\left(z_{1}, z_{2}, z_{3}, z_{4}\right)$ in $\Omega$ consists of a Jordan domain $\Omega$ together with four positively oriented vertices $z_{1}, z_{2}, z_{3}, z_{4} \in \partial \Omega$ which divides $\partial \Omega$ into four sides. Then $Q$ can be mapped conformally onto a rectangle $R=R(0, m, m+i, i)$ so that the vertices and sides of $Q$ and $R$ correspond. The modulus of $Q$ is defined as $\bmod (Q)=m$. The conjugate quadrilateral of $Q$ is defined as the quadrilateral $Q^{*}=\Omega^{*}\left(z_{4}, z_{3}, z_{2}, z_{1}\right)$ in $\Omega^{*}$. It is easy to see that $\bmod (Q)$ is also the modulus of the curve family that join the opposite sides from $z_{1}$ to $z_{2}$ and from $z_{3}$ to $z_{4}$ in $\Omega$.

4.1. Quadrilateral inequality. We say that $\Omega$ has this property if there is a constant $c \geq 1$ such that

$$
\frac{1}{c} \leq \frac{\bmod (Q)}{\bmod \left(Q^{*}\right)} \leq c
$$

for all conjugate quadrilaterals $Q$ and $Q^{*}$ in $\Omega$ and $\Omega^{*}$, respectively.

4.2. Extremal distance property. We say that $\Omega$ has this property if there is a constant $c \geq 2$ such that

$$
\bmod (A, B ; \mathbb{C}) \leq c \bmod (A, B ; \Omega)
$$

for any pair of disjoint continua $A, B \subset \partial \Omega$.

It is well known that $\partial \Omega$ is a quasicircle if and only if it satisfies the quadrilateral inequality and if and only if it has the extremal distance property (see [Ge, Theorem III.D. 4 and Theorem III.E.4], or [LV, GM]). It is also known that $\Omega$ is a disk or half plane if and only if it satisfies the quadrilateral inequality with $c=1$ and if and only if it has the extremal distance property with $c=2$, [Y1]. Similar to the strong reverse triangle inequality introduced by Pommerenke, we introduce the strong versions of the above properties and use them to characterize symmetric quasicircles.

4.3. Strong quadrilateral inequality. We say that $\Omega$ has this property if for any $\epsilon>0$ there exists $\delta>0$ such that

$$
\frac{1}{1+\epsilon} \leq \frac{\bmod (Q)}{\bmod \left(Q^{*}\right)} \leq 1+\epsilon
$$

for all conjugate quadrilaterals $Q$ and $Q^{*}$ in $\Omega$ and $\Omega^{*}$ with one of the sides of $Q$ having diameter less than $\delta$. 
4.4. Strong extremal distance property. We say that $\Omega$ has this property if for any $\epsilon>0$ there exists $\delta>0$ such that

$$
\bmod (A, B ; \mathbb{C}) \leq(2+\epsilon) \bmod (A, B ; \Omega)
$$

for all continua $A, B \subset \partial \Omega$ with $\min \{\operatorname{diam}(A), \operatorname{diam}(B)\}<\delta$.

THEOREM 4.1. For a Jordan domain $\Omega$, the following statements are equivalent.

(a) $\partial \Omega$ is a symmetric quasicircle;

(b) $\Omega$ has the strong extremal distance property;

(c) $\Omega$ satisfies the strong quadrilateral inequality.

PROOF. We first show that (a) implies (b). Suppose that $\partial \Omega$ is a symmetric quasicircle. To show that $\Omega$ has the strong extremal distance property, we assume the contrary. Then there exist $\epsilon_{0}>0$ and disjoint continua $A_{n}, B_{n} \in \partial \Omega(n=1,2, \ldots)$ such that

$$
\frac{\bmod \left(A_{n}, B_{n} ; \mathbb{C}\right)}{\bmod \left(A_{n}, B_{n} ; \Omega\right)} \geq 2+\epsilon_{0}
$$

for all $n \geq 1$ and such that $A_{n}$ and $B_{n}$ converge in the Hausdorff metric to continua $A$ and $B$, respectively, where $A$ is a single point. Then the argument above for Case 2 and Case 3 in the proof of Theorem 2.1 shows that

$$
\varlimsup_{n \rightarrow \infty} \frac{\bmod \left(A_{n}, B_{n} ; \mathbb{C}\right)}{\bmod \left(A_{n}, B_{n} ; \Omega\right)} \leq 2 H,
$$

where $H$ is the local reflection constant of $\partial \Omega$. Since $\partial \Omega$ is a symmetric quasicircle, $H=1$ by [GS, Proposition 3.2]. Therefore (4.6) contradicts (4.5). This proves that $\Omega$ has the strong extremal distance property as desired.

Next we show that (b) implies (c). Assume that $\Omega$ has the strong extremal distance property. For any $\epsilon>0$ let $\delta>0$ be as in (4.4). Let $Q=\Omega\left(z_{1}, z_{2}, z_{3}, z_{4}\right)$ be any quadrilateral in $\Omega$ with one of its sides, say the one from $z_{1}$ to $z_{2}$, having diameter less that $\delta$. Denote this side by $A$ and its opposite side by $B$. Then it is easy to see that

$$
\bmod (Q)=\bmod (A, B ; \Omega), \quad \bmod \left(Q^{*}\right)=\bmod \left(A, B ; \Omega^{*}\right),
$$

where $Q^{*}$ is the conjugate quadrilateral of $Q$ in $\Omega^{*}$. By the monotonicity of the modulus and by the strong extremal distance property, it follows that

$$
\frac{\bmod (A, B ; \Omega)+\bmod \left(A, B ; \Omega^{*}\right)}{\bmod (A, B ; \Omega)} \leq \frac{\bmod (A, B ; \mathbb{C})}{\bmod (A, B ; \Omega)} \leq 2+\epsilon
$$


This yields that

$$
\frac{\bmod \left(Q^{*}\right)}{\bmod (Q)}=\frac{\bmod \left(A, B ; \Omega^{*}\right)}{\bmod (A, B ; \Omega)} \leq 1+\epsilon
$$

Thus the strong quadrilateral inequality (4.3) follows by symmetry.

Finally, we show that (c) implies (a). Assume that $\Omega$ satisfies the strong quadrilateral inequality. Let $h$ be a homeomorphism of the unit circle $\partial D$ induced by $\Omega$. Then the strong quadrilateral inequality for $\Omega$ implies that for any $\epsilon>0$ there exists a $\delta>0$ such that

$$
\frac{1}{1+\epsilon} \leq \frac{\bmod (h(A), h(B) ; D)}{\bmod (A, B ; D)} \leq 1+\epsilon
$$

for any pair of disjoint nondegenerate continua $A$ and $B$ on the unit circle with $\min \{\operatorname{diam}(A), \operatorname{diam}(B)\}<\delta$.

We shall show that $h$ satisfies the strong $M$-condition (1.3). Suppose otherwise. Then there exist $\epsilon_{0}>0$ and positively oriented points $a_{n}, b_{n}, c_{n} \in \partial D(n \geq 1)$ with $\left|a_{n}-b_{n}\right|=\left|b_{n}-c_{n}\right| \leq 1 / n$ such that

$$
\frac{\left|h\left(c_{n}\right)-h\left(b_{n}\right)\right|}{\left|h\left(b_{n}\right)-h\left(a_{n}\right)\right|}>1+\epsilon_{0}
$$

for all $n \geq 1$. Denote the opposite point of $b_{n}$ on the circle by $d_{n}$ and denote the images of $a_{n}, b_{n}, c_{n}, d_{n}$ under $h$ by $a_{n}^{\prime}, b_{n}^{\prime}, c_{n}^{\prime}, d_{n}^{\prime}$, respectively. By passing to subsequences, we may assume that $a_{n}, b_{n}, c_{n} \rightarrow a$ and $d_{n} \rightarrow d$ as $n \rightarrow \infty$. Furthermore, let $A_{n}$ denote the closed arc from $a_{n}$ to $b_{n}$ and $B_{n}$ the closed arc from $c_{n}$ to $d_{n}$. Using some basic properties for the modulus, one can deduce that

$$
\frac{\bmod \left(h\left(A_{n}\right), h\left(B_{n}\right) ; D\right)}{\bmod \left(A_{n}, B_{n} ; D\right)}=\frac{\log \Psi\left(\left[a_{n}, b_{n}, c_{n}, d_{n}\right]\right)}{\log \Psi\left(\left[a_{n}^{\prime}, b_{n}^{\prime}, c_{n}^{\prime}, d_{n}^{\prime}\right]\right)},
$$

where $\Psi:(0, \infty) \rightarrow(1, \infty)$ is a strictly increasing function determined by the Teichmüller ring domain and $[a, b, c, d]$ denotes the absolute cross ratio:

$$
[a, b, c, d]=\frac{|a-d||b-c|}{|a-b||c-d|} .
$$

Since $\left[a_{n}, b_{n}, c_{n}, d_{n}\right]=1$ for all $n \geq 1$ and $\left|d_{n}^{\prime}-a_{n}^{\prime}\right| /\left|d_{n}^{\prime}-c_{n}^{\prime}\right| \rightarrow 1$ as $n \rightarrow \infty$, it follows from (4.8) and (4.10) that

$$
\frac{1}{1+\epsilon^{\prime}} \leq \frac{\left|c_{n}^{\prime}-b_{n}^{\prime}\right|}{\left|b_{n}^{\prime}-a_{n}^{\prime}\right|} \leq 1+\epsilon^{\prime},
$$

where $\epsilon^{\prime}=\epsilon^{\prime}(n) \rightarrow 0$ as $n \rightarrow \infty$. This contradicts (4.9) and shows that $h$ satisfies a strong $M$-condition on the unit circle. Hence $h$ is symmetric and $\partial \Omega$ is a symmetric quasicircle. This completes the proof of Theorem 4.1. 
REMARK. According to the strong quadrilateral inequality characterization, we can make the following comparison between symmetric and non-symmetric quasicircles. Let us consider the ratio of the extremal distances between any two continua $A$ and $B$ on a Jordan curve when seen from inside and outside. Then at large scales this ratio is bounded for both symmetric and non-symmetric quasicircles. However, at arbitrarily small scales, this ratio is bounded by the same finite constant for a non-symmetric quasicircle, whereas this ratio gets closer and closer to one for a symmetric quasicircle as the scale gets smaller. As a corollary to this result, we finally derive a similar characterization for symmetric homeomorphisms.

COROLLARY 4.2. Let h be a homeomorphism of the unit circle. Then $h$ is symmetric if and only if it satisfies the strong quadrilateral inequality: For any $\epsilon>0$ there exists $\delta>0$ such that

$$
\frac{1}{1+\epsilon} \leq \frac{\bmod (Q)}{\bmod (h(Q))} \leq 1+\epsilon
$$

for any quadrilateral $Q=D\left(z_{1}, z_{2}, z_{3}, z_{4}\right)$ in the unit disk with one of its sides having diameter less than $\delta$.

PROOF. The sufficiency has been proved in Theorem 4.1 (see the part (c) implies (a)). For the necessity, we observe that the argument for the degenerate case in the proof of [Wu, Theorem 1] shows that

$$
\varlimsup_{\delta \rightarrow 0} \sup \frac{\bmod (Q)}{\bmod (h(Q))} \leq H,
$$

where $H$ is the boundary dilatation of $h$ and where the supremum is taken over all quadrilaterals $Q$ in $D$ with one of its sides having diameter less than $\delta$. Once again $h$ is symmetric implies that $H=1$. Thus the strong quadrilateral inequality (4.11) follows from (4.12).

\section{References}

[Ba] T. Bagby, 'The modulus of a plane condenser', J. Math. Mech. 17 (1967), 315-329.

[BP] J. Becker and C. Pommerenke, 'Über die quasikonforme Fortsetzung schlichter Funktionen', Math. Z. 161 (1978), 69-80.

[Fe] R. Fehlmann, 'Über extremale quasikonforme Abbidungen', Comment. Math. Helv. 56 (1981), 558-580.

[Ga] F. P. Gardiner, Teichmüller theory and quadratic differentials (Wiley, New York, 1987).

[GS] F. P. Gardiner and D. P. Sullivan, 'Symmetric structures on a closed curve', Amer. J. Math. 114 (1992), 683-736. 
[GY] J. B. Garnett and S. Yang, 'Quasiextremal distance domains and integrability of derivatives of conformal mappings', Michigan Math. J. 41 (1994), 389-406.

[Ge] F. W. Gehring, 'Characterizations of quasidisks', preprint (1997).

[GM] F. W. Gehring and O. Martio, 'Quasiextremal distance domains and extension of quasiconformal mappings', J. Anal. Math. 45 (1985), 181-206.

[He] J. Hesse, 'A p-extremal length and p-capacity equality', Ark. Mat. 13 (1975), 131-144.

[Ku] R. Kühnau, 'Möglichst konforme Spiegelung an einer Jordankurve', Jber. d. Dt. Math. Verein. 90 (1988), 90-109.

[LV] O. Lehto and K. I. Virtanen, Quasiconformal mappings in the plane (Springer, New York, 1973).

[Po] C. Pommerenke, Boundary behaviour of conformal maps (Springer, Berlin, 1992).

[Wu] S. Wu, Moduli of quadrilaterals and extremal quasiconformal extensions of quasisymmetric functions', Comment. Math. Helv. 72 (1997), 593-604.

[Y1] S. Yang, 'QED domains and NED sets in $R^{n}$ ', Trans. Amer. Math. Soc. 334 (1992), 97-120.

[Y2] - 'On dilatations and substantial boundary points of homeomorphisms of Jordan curves', Result. Math. 31 (1997), 180-188.

[Y3] - 'A modulus inequality for condensers and conformal invariants of smooth Jordan domains', J. Anal. Math. 75 (1998), 173-183.

Department of Mathematics

Peking University

Beijing 100871

China

e-mail: wusj@pku.edu.cn
Department of Mathematics and Computer Science Emory University Atlanta GA 30322

USA

e-mail: syang@mathcs.emory.edu 